\title{
LA NECESARIA FORMACIÓN DEL PROFESORADO UNIVERSITARIO ANTE LA DOCENCIA VIRTUAL
}

The necessary training of university teachers in the face of virtual teaching

Recibido: 3 de octubre de 2020

Aceptado: 14 de diciembre de 2021

Esther Rando Burgos

Profesora de Derecho Administrativo

(acreditada Profesora Contratada Doctora)

erando@uma.es

Universidad de Málaga

\section{RESUMEN}

Los últimos meses, con la fatídica llegada de la Covid-19, han supuesto un auténtico tsunami en nuestras vidas, en todas las esferas, también en la docente. En apenas días se pasó de la ya tradicional docencia presencial a la docencia virtual. Estudiantes $\mathrm{y}$ docentes nos vimos de lleno en un nuevo escenario que hasta entonces se limitaba a cuestiones concretas y que transformó nuestro día a día. Y, podría afirmarse, que se ha superado con éxito o, al menos, de la mejor forma posible dado los limitados recursos, la situación de incertidumbre generalizada, gracias a una reinvención casi diaria. Transcurridos estos primeros tiempos y aún sin saber qué escenario tendremos en el próximo curso una cuestión parece evidente: la docencia virtual ha llegado para quedarse. Consecuencia de ello es la necesaria adaptación del docente al nuevo escenario, lo que pasa por la formación en docencia virtual para, valga la redundancia, poder formar a nuestros estudiantes. El objetivo del presente trabajo se centra en analizar la importancia y necesaria formación del docente en el entorno universitario ante la docencia virtual.

\section{PALABRAS CLAVE}

formación docente, docencia virtual, estudiante, enseñanzas universitarias.

\begin{abstract}
The last few months, with the fateful arrival of Covid-19, have been a real tsunami in our lives, in all spheres, also in teaching. In just days he went from traditional face-to-face teaching to virtual teaching. Students and teachers we saw each other in a new scenario that until then was limited to concrete issues and that became our day to day. And, it could be said, that it has been successfully overcome, or at least in the best possible way given the limited resources, the situation of widespread uncertainty, thanks to an almost daily reinvention. After these early days and still not knowing what scenario we will have in the next course a question seems obvious: virtual teaching is here to stay. As a result, the necessary adaptation of the teacher to the new scenario, which goes through the training in virtual teaching for, worth the redundancy, to be able to train our students. The objective of this work focuses on analyzing the importance and necessary training of the teacher in the university environment in the face of virtual teaching.
\end{abstract}

\section{KEYWORDS}

teacher training, virtual teaching, student, university teaching. 
Sumario: 1. Introducción. 2. La formación docente. 3. El profesorado universitario ante la docencia virtual. 4. Otros aspectos esenciales a tener en cuenta para una docencia universitaria virtual de calidad. 5. Reflexiones. 6. Bibliografía.

\section{Introducción.}

La formación del docente constituye una herramienta esencial y continuada en el tiempo en lo relativo al proceso de docencia. Las propias Universidades llevan a cabo planes de formación destinados precisamente a ofrecer a los docentes esa labor y actualización que requiere un adecuado ejercicio de la docencia. No sólo en el ámbito propio de lo que comúnmente denominamos formación docente, también en otros ámbitos estrechamente relacionados con el mismo, como formación en idiomas, formación en TIC, formación para la investigación o formación trasversal, entre otras.

El docente en su labor de enseñanza debe estar provistos no sólo de los conocimientos propios de la materia de su especialidad, sino complementarlos con un conjunto de herramientas destinadas a hacer de esa enseñanza un proceso de aprendizaje. Es esencial esa adecuada transmisión de conocimientos al estudiante y aquí juega un papel relevante la mejora continua de la actividad docente, desarrollada paralelamente a aquélla, y en la que el docente pasa del rol habitual de formador a estudiante, valga la redundancia, con el claro objetivo de tener las habilidades y herramientas necesarias para poder formar mejor a sus estudiantes,

Cursos, jornadas, congresos, seminarios o talleres destinados a la actividad docente, se combinan en la labor diaria del mismo. Hasta aquí ninguna novedad.

Sin embargo, esta formación en docencia, habitual en las Universidades y a la que con mayor o menor frecuencia acudíamos los docentes, e importante como se indicaba, se ha transformado en una auténtica necesidad para poder transitar en el marco en el que nos vemos inmersos en la actualidad: el tránsito de la docencia presencial a la docencia virtual, con una vez cada más prevalencia de la segunda. Cierto es que la docencia virtual no representaba ya un hecho desconocido, desde tiempo atrás es una realidad, incluso en el proceso de formación presencial cada vez representaba una parte más importante, eso sí, casi limitada en la práctica y en lo relativo a las enseñanzas oficiales universitarias, al uso de los conocidos como "campus virtual", como complemento de la docencia. Hasta ahora, el campus virtual casi tenía más una función de "foro de encuentro" en el que unificar la información relativa a las diferentes asignaturas, lugar de entrega de actividades o trabajos, o incluso como medio de comunicación de mensajería entre estudiantes y docentes.

La llegada de la Covid-19, transformó radicalmente, en apenas días, el protagonismo del campus virtual, pasando a "convertirse" en las "aulas" en las que, junto a los anteriores, se ha venido desarrollando toda la labor docente, incluidas las tradicionales "sesiones magistrales" reconvertidas en videoconferencias.

Esta nueva situación, aún muy someramente expuesta, ha obligado a los docentes a reinventarse casi a diario, adaptando, reformulando y volviendo a reformular, indagando en la búsqueda continua de nuevos y más efectivos recursos, todo con una meta clara: ofrecer la mejor docencia a nuestros estudiantes y que el obligado cambio o trasvase de docencia presencial a docencia virtual, conllevase en la práctica el menor impacto en aquéllos, tratando de minimizar los efectos e incluso percepción que sobre ellos pudiera tener. 


\section{La formación docente.}

El docente tiene la constante necesidad de adaptarse a entornos cada vez más cambiantes. La sociedad del conocimiento, en la que nos encontramos docentes y estudiantes, exige el desarrollo de multitud de competencias a desarrollar y para las que se han de adquirir las habilidades necesarias de cara a ampliar y diversificar el proceso de aprendizaje.

La propia Ley Orgánica 6/2001, de 21 de diciembre, de Universidades (LOU), destaca la función clave de las Universidades en la sociedad actual, reconociendo su papel central en el desarrollo cultural, económico y social del país, debiendo responder al dinamismo de una sociedad avanzada, pero, además, haciéndolo

«... de forma coherente y global, debe sistematizar y actualizar los múltiples aspectos académicos, de docencia, de investigación y de gestión, que permitan a las Universidades abordar, en el marco de la sociedad de la información y el conocimiento, los retos derivados de la innovación en las formas de generación y transmisión del conocimiento».

Este papel central de las Universidades en el desarrollo cultural, económico y social de un país plantea como reto que la sociedad podrá exigir de sus Universidades las más valiosas de las herencias para su futuro: una docencia de calidad, una investigación de excelencia.

Lograr dicha docencia de calidad se plantea en un escenario en el que la formación y el conocimiento son factores claves, sometidos a vertiginosas transformaciones sociales y económicas. Pero, además, con el compromiso que para las Universidades implica las demandas que la nueva sociedad requiere: profesionales con un elevado nivel cultural, científico y técnico que la enseñanza universitaria debe proporcionar. Sociedad y Universidad quedan así estrecha y recíprocamente unidas. En congruencia con lo anterior, cuando la LOU, en su artículo 2, "Autonomía universitaria», desarrolla qué ha de entenderse por dicha autonomía de las Universidades, se refiere expresamente, entre otros, a «la selección, formación y promoción del personal docente e investigador y de administración y servicios, así como la determinación de las condiciones en que han de desarrollar sus actividades» (art. 2.2.e LOU).

Cuestión que se reitera en el articulado de la norma, entre otros, en el artículo 33 «e la función docente», cuando establece:

1. Las enseñanzas para el ejercicio de profesiones que requieren conocimientos científicos, técnicos o artísticos, y la transmisión de la cultura son misiones esenciales de la Universidad.

2. La docencia es un derecho y un deber de los profesores de las Universidades que ejercerán con libertad de cátedra, sin más límites que los establecidos en la Constitución y en las leyes y los derivados de la organización de las enseñanzas en sus Universidades.

3. La actividad y la dedicación docente, así como la formación del personal docente de las Universidades, serán criterios relevantes, atendida su oportuna evaluación, para determinar su eficiencia en el desarrollo de su actividad profesional.

Formación docente que se convierte así en criterio relevante para determinar la eficiencia en el desarrollo de la propia actividad profesional. La integración en el Espacio Europeo de Integración Superior (EEES) conllevó una profunda reforma no sólo en la estructura organizativa de la Universidad sino en la formulación de los retos a afrontar por 
la enseñanza. Como señalan Calderón Patier y González Lorente, en este nuevo contexto, el profesor universitario debe simultanear su labor docente e investigadora (dos actividades complementarias), pero a su vez, y junto a esa transmisión de conocimientos, debe también transmitir valores, habilidades y actitudes que contribuyan a la formación integral de los estudiantes. Sin embargo, la cuestión va mucho más allá, y en este escenario no debe obviarse, como también destacan, que «El EEES no es una idea aislada de un grupo de políticos europeos, sino que responde a la necesidad de cambiar la Universidad europea para dar respuesta a la nueva situación económica, política, social, laboral, cultural e incluso geográfica existente, que viene determinada principalmente por la revolución tecnológica. Las nuevas tecnologías de la información y la comunicación han transformado la sociedad y han permitido, no sólo una difusión y acceso generalizado a la información, sino también, y más importante aún, una necesaria revisión, cambio e incorporación de las mismas en los procesos educativos, tanto en su ámbito formativo como investigador. Y es aquí donde aparece un nuevo y fundamental rol o papel del profesor universitario: el profesor debe pasar a ser el enseñante del aprendizaje del alumno, es decir, será la guía de los alumnos que les facilite y les enseñe el adecuado uso de los recursos disponibles e instrumentos que necesiten, esto es, es un gestor del aprendizaje» ${ }^{1}$.

Este contexto nos lleva indudablemente a destacar la importancia de la continua formación de los docentes universitarios. Sin embargo, y aquí el interés del presente trabajo, esta importancia se ha transformado casi de repente en una necesidad acuciante. $\mathrm{O}$ se apuesta de manera urgente por la puesta al día en docencia virtual o difícilmente se podrá continuar prestando adecuadamente la labor de aprendizaje que como docentes tenemos atribuida.

\section{El profesorado universitario ante la docencia virtual.}

La transformación digital del proceso de enseñanza en la formación universitaria, tanto en su modalidad semipresencial como en la modalidad a distancia (completamente on line), requieren una innovación didáctica del docente universitario dirigida a la adquisición de las competencias y metodologías que posibiliten adquirir los conocimientos orientados a una reformulación en el modelo, hasta ahora tradicional, de enseñanza y aprendizaje, tradicional al virtual.

Su consecución pasa necesariamente por la formación del profesorado universitario en aras a adquirir y mejorar de manera continua la docencia y los resultados de aprendizaje de los estudiantes.

Es cierto que con la llegada de la Covid-19 ha pasado de ser opcional y convivir con la docencia presencial, a instaurarse en una nueva necesidad. La formación de los estudiantes en general y de los universitarios en particular, no sólo es un derecho fundamental recogido como tal en el artículo 27 de la Constitución Española, debe ser una prioridad a la que las diferentes Administraciones deben dar la adecuada respuesta, sin obviar su adecuada articulación, en momentos como el actual de pandemia a nivel mundial, con la salud y la vida. Y en este escenario, la docencia virtual, con sus ventajas e inconvenientes, se presenta como una de las escasas alternativas a la docencia presencial.

\footnotetext{
${ }^{1}$ CALDERÓN PATIER, C. y GONZÁLEZ LORENTE, Á. (2009) «El papel del profesor universitario español en el EEES». Disponible: https://www.uv.es/motiva2/Ponencias\%20Motiva2009/docs/53.pdf [última consulta 17/07/2020].
} 
Sin embargo, no se está afortunadamente ante algo desconocido. Todo lo contrario, la docencia semipresencial y on line llevan años de andadura.

Una de las cuestiones esenciales es ofrecer una docencia universitaria virtual de calidad. Ello implica que, a las tradicionales tareas investigadoras, docentes y de gestión que habitualmente ocupan a los profesores universitarios, se une una cuarta y fundamental: su propia formación. No es suficiente con las competencias y conocimientos tradicionales, se adiciona un nuevo conjunto de competencias que el docente debe manejar y que se convierten así en nexo preciso a conjugar para lograr una docencia universitaria de calidad. Estas competencias precisas en la docencia virtual están a su vez conformadas por diferentes aspectos, todos ellos estrechamente relacionados y que deberán ser conjugados en la práctica. Autores como Durán Rodríguez y Estay-Niculcar ${ }^{2}$, al analizar la importancia de seleccionar un modelo de buenas prácticas para la educación virtual, incluyen cuatro componentes: el aula virtual, la biblioteca digital, los tutores virtuales y el campus virtual. Destacan para cada uno de ellos «... (1) el aula virtual, la cual es una adaptación del aula tradicional de clases con la inclusión de componentes tecnológicos avanzados ${ }^{3}$; (2) la biblioteca, que es una extensión de la biblioteca tradicional con la capacidad de manejar altos volúmenes de información ${ }^{4}$; (3) los tutores virtuales, quienes requieren las mismas competencias del docente tradicional y en adición un nuevo conjunto de competencias informacionales ${ }^{5}$; y (4) el campus virtual, que es una extensión del campus universitario tradicional donde el estudiante puede acceder; a través de un ordenador con conexión a Internet; a los servicios administrativos y académicos de la Universidad tales como: gestionar matrícula, realizar pagos, consultar calificaciones, solicitar el último informe de calificaciones, gestionar un documento de paz y salvo, entre otros ${ }^{6} \gg$.

Por todo ello, los docentes universitarios nos enfrentamos a un nuevo reto, como se ha señalado con anterioridad, un reto que si bien antes ya existía y sobre el que se tenían habilidades y competencias ya adquiridas hoy se presenta como una necesidad. Que, además, como se apuntaba, bajo la casi genérica frase «docencia virtual» se engloba diferentes cuestiones que hacen imprescindible la formación en diferentes ámbitos. No parece suficiente con el manejo de Moodle, la plataforma educativa que da soporte a buena parte de los campos virtuales, es imprescindible desenvolverse en todas y las múltiples posibilidades que maneja la misma lo que en ocasiones llevará a precisar de una previa formación sobre su utilización para poder usar y aprovechar todos los recursos que la misma ofrece ${ }^{7}$. Como apuntan Sánchez Santamaría y Morales Calvo «... el simple hecho de hacer uso de Moodle u otros sistemas basados en LMS no conlleva una innovación o

\footnotetext{
2 DURÁN RODRÍGUEZ, R. y ESTAY-NICULCAR, C.A. (2016) «Las buenas prácticas docentes en la educación virtual universitaria». REDU, Revista de docencia universitaria, vol. 14 (2), julio-diciembre, págs. 159-186. Disponible: https://polipapers.upv.es/index.php/REDU/issue/view/638 [última consulta 30/07/2020].

${ }^{3}$ Como señalan acogiendo la idea de TINTAYA, A.E. (2009) Desafíos y fundamentos de educación virtual. Buenos Aires, El Cid Editor.

${ }^{4}$ Conforme a la referencia de los autores, en VARELA-OROL, C. (2011) « ¿Hacia un nuevo paradigma bibliotecario? El nuevo orden digital». El profesional de la información, 20 (5), págs. 564-570.

${ }^{5}$ Por remisión de los autores, en GARCÍA ARETIO, L. (2013) «Fundamento y Componentes de la Educación a Distancia». RIED. Revista Iberoamericana de educación a distancia, 2 (2).

${ }^{6}$ Conforme a la cita de referencia, en FROILÁN, J. Y GISBERT, M. (2012) «El cambio organizacional en la universidad a través del uso de los campus virtuales desde la perspectiva de los estudiantes». Pixel-Bit, Revista de Medios y Educación, núm. 40, págs. 75-88.

${ }^{7}$ Sobre la evolución de los campus virtuales, veáse, entre otros, URBINA, S. y SALINAS, J. (2014) «Campus virtuales: una perspectiva educativa y tendencias». RED. Revista de Educación a Distancia, núm. 42, septiembre-diciembre, págs. 6-21. Disponible: https://www.redalyc.org/pdf/547/54731828002.pdf [última consulta 30/07/2020].
} 
mejora de los procesos de enseñanza-aprendizaje, ya que el aprendizaje on-line requiere de unas condiciones y recursos adecuados vinculados al diseño, contenido, desarrollo, herramientas de trabajo, apoyos del profesorado, percepciones de los alumnos, experiencias previas, entre otras» ${ }^{8}$. Es preciso, en el escenario actual, ese tránsito en el que el espacio virtual se convierte en auténtico protagonista, reforzando la función que ha desempeñado hasta el momento y que, como ya se ha señalado, constituía una realidad con una importante implantación. En este sentido, Area Moreira, San Nicolás Santos y Sanabria Mesa, señalan que «En las universidades presenciales, entre las múltiples formas, modalidades y estrategias de uso pedagógico de las tecnologías digitales (MOOC, flipped classroom, realidad aumentada, PLE, microcursos online, entornos adaptativos, analíticas del aprendizaje,...), la docencia presencial apoyada en un aula virtual dentro de un campus digital institucional es la experiencia y práctica más generalizada entre el profesorado y el alumnado en las titulaciones oficiales tanto de grado como postgrado» ${ }^{9}$, a lo que añaden un dato revelador, así los autores, citando a Piriz Durán ${ }^{10}$, recuerdan como en «La Conferencia de Rectores de Universidades Españolas, en el informe UNIVERSITIC 2015, señalaba que desde el año 2013 más del 90\% del profesorado de las universidades utilizaba la plataforma de docencia virtual institucional, lo que constituye una importante consolidación y generalización de esta modalidad educativa».

Sin embargo, no es lo mismo el empleo de estos recursos como apoyo a la docencia presencial o su utilización como complemento o refuerzo de la misma, que su consolidación como medio prioritario o exclusivo para la impartición de la referida docencia universitaria. Este hecho justifica la necesidad ya expuesta de una formación adecuada y trasversal del docente universitario no ya sólo en su manejo sino en la utilización de todos cuantos recursos y posibilidades ofrece la misma ${ }^{11}$.

\section{Otros aspectos esenciales a tener en cuenta para una docencia universitaria virtual de calidad.}

Como venimos señalando, la cuestión referida al logro de una docencia virtual universitaria de calidad exige muchos otros aspectos. El propio modo de impartir las tradicionales clases magistrales también se ve afectado de manera exponencial, lo que hará preciso su rearticulación en aras a, como se sostiene, mantener la excelencia en la docencia universitaria. A la característica lección magistral le sustituye a día de hoy la lección virtual. El contexto, en el que se pasa del aula tradicional al aula virtual, en el que ya no se da esa interacción personal con los estudiantes y que se instituye casi en esencial de la

\footnotetext{
${ }^{8}$ SÁNCHEZ SANTAMARÍA, J. Y MORALES CALVO, S. (2012) «Docencia universitaria con apoyo en entornos virtuales de aprendizaje (EVA)». Digital Education Review, núm. 21, págs. 33-46 (pág. 36). Disponible: http://greav.ub.edu/der/ [última consulta 02/09/2020].

${ }^{9}$ AREA MOREIRA, M., SAN NICOLÁS SANTOS, M.B. Y SANABRIA MESA, L. (2018) «Las aulas virtuales en la docencia de una universidad presencial: la visión del alumnado». Revista Iberoamericana de Educación a Distancia, 21(2), págs. 179-198 (pág. 180). Disponible: http://dx.doi.org/10.6018/red/56/1 [última consulta 03/09/2020].

${ }^{10}$ PIRIZ DURÁN, S. (2015) UNIVERSITIC 2015. Análisis de las TIC en las Universidades Españolas. Madrid: Crue Universidades Españolas. Disponible: http://www.crue.org/Documentos\%20 [última consulta 03/09/2020].

${ }^{11}$ Sobre el proceso y evolución del docente en relación a la innovación de la práctica de la enseñanza universitaria mediante el empleo de internet, resulta de interés el trabajo de AREA MOREIRA en el que narra en primera persona su propia historia a lo largo de veinte años, como señala, «... está narrada como una historia de vida basada en los distintos "selfies profesionales" que fui recogiendo a lo largo mi experiencia de docente con las tecnologías digitales». En AREA MOREIRA, M. (2018) «De la enseñanza presencial a la docencia digital. Autobiografía de una historia de vida docente». RED. Revista de Educación a Distancia, núm. 56, art.1, págs. 1-21. Disponible: http://dx.doi.org/10.6018/red/56/1 [última consulta 05/09/2020].
} 
docencia presencial y una de las cuestiones que más se achaca de ésta frente a la virtual. Cuestiones como las anteriores nos lleva a que no todos los conocimientos precisos para la docencia virtual pueden ser adquiridos mediante la formación del docente y hay cuestiones que inevitablemente serán, cuando menos, diferentes.

Aquí juega, nuevamente, un papel fundamental el rol que asuma el docente, en la medida en que entran en juego otros aspectos, como la necesaria motivación del estudiante, el seguimiento del mismo, el acompañamiento y la empatía, en otros tantos que se podrían señalar. Surgen aspectos que en la docencia presencial tienen menor incidencia y que por ende, precisan su adecuada atención. Tratar de prevenir el abandono de la formación, lograr el compromiso e interés de los estudiantes con la formación universitaria virtual, una adecuada transmisión del conocimiento o la propia adaptación en el diseño de la evaluación de los aprendizajes, son sólo algunas cuestiones que se entiende requieren particular consideración.

El tema de la motivación se entiende esencial en un escenario de enseñanza virtual, como medio para trata de, en cierta medida, paliar la carencia de esa cercanía que sí tiene lugar en la docencia presencial. Precisamente en una de las variables que con mayor frecuencia se producen en la docencia virtual, el abandono del estudiante, la motivación se instituye en un factor clave. Autores como García Aretio, al analizar la problemática del abandono del estudiante, se pregunta cuáles deberán ser las preocupaciones institucionales y docentes para dar respuesta al problema del abandono, al margen de otras causas también presentes como los factores socioeconómicos de la situación personal, familiar y contextual del estudiante, más difícilmente alterables desde la perspectiva institucional o docente. Entre las respuestas desde la institución, el autor señala que debe atenderse de manera particular a cinco aspectos: respecto a la falta de tiempo, técnicas de estudio; la motivación; la escasa información y orientación inicial; el abandono temprano, la integración académica y social, cursos cero y de acogida; la apuesta tecnológica. Como respuestas desde la docencia, lo focaliza en cuatro cuestiones: elaborar la guía de estudio; diseñar, seleccionar y/o elaborar los diferentes materiales de estudios; gestionar la actividad docente; gestionar la actividad docente; activar los diferentes modelos y formas de evaluar $^{12}$.

Todo ello, sin obviar las posibilidades que esta modalidad ofrece, de ahí la necesidad de que el profesorado universitario esté dotado de las herramientas, mediante el aprendizaje en docencia virtual, para poder implementarlo y reconvertirlo en una oportunidad. En este sentido, Tony Bates ${ }^{13}$ destaca que la opción de optar por una modalidad semipresencial, híbrida u online y de optar por un mayor uso de las tecnologías de aprendizaje ofrece más alternativas a los profesores e instructores, pero para implementar adecuadamente estas tecnologías, el profesorado no sólo debe identificar sus fortalezas y debilidades sino que también debe contar con un buen conocimiento de cómo aprenden mejor los estudiantes. A su vez, identifica tres que es preciso conocer: las investigaciones sobre la enseñanza y el aprendizaje; diferentes teorías del aprendizaje relacionadas con diferentes tipos de conocimiento; diferentes métodos de enseñanza y sus debilidades y fortalezas, recordando que sin ese punto de partida, o fundamento básico, en palabras del autor, es difícil para los profesores alejarse del único modelo con el que están familiarizados: la clase magistral y

${ }^{12}$ GARCÍA ARETIO, L. (2019) «El problema del abandono en estudios a distancias. Respuestas desde el Diálogo Didáctico Mediano». RIED, Revista Iberoamericana de Educación a Distancia, Asociación Iberoamericana de Educación Superior a Distancia, vol. 22, núm. 1. Disponible: https://doi.org/10.5944/ried.22.1.22433 [última consulta 06/09/2020].

${ }^{13}$ TONY BATES, A.W. (2017) La enseñanza en la Era Digital. Una guía para la enseñanza y el aprendizaje. Obra publicada bajo licencia internacional de propiedad Creative Commnos no comercial. 
el modelo de debate, limitado en cuanto al desarrollo de los conocimientos y las competencias necesarias en la era digital.

Y este escenario que expone de manera general, se agrava, como también señala en las Universidades, «...ya que no hay ningún requisito de formación docente para trabajar en una universidad en la mayoría de los países occidentales. Sin embargo, la enseñanza ocupará un mínimo del $40 \%$ del tiempo del profesor experto y mucho más para los profesores adjuntos o contratados o los instructores de dedicación exclusiva. No obstante, en menor grado, los maestros de escuela y los profesores universitarios enfrentan el mismo reto: cómo garantizar que los profesionales con experiencia dominen los conocimientos y competencias necesarias para enseñar bien en la era digital». Y entiende que es aquí donde son las instituciones las protagonistas «para facilitar o impedir el desarrollo de los conocimientos y competencias para la era digital» para lo que plantea, a modo de propuestas: asegurar que el personal de todos los niveles de enseñanza e instrucción tengan la capacitación adecuada en el uso de las nuevas tecnologías y los métodos de enseñanza necesarios para el desarrollo del conocimiento y las competencias en la era digital; asegurar que se ofrezca el apoyo adecuado a los profesores en el uso de las tecnologías educativas; asegurar que las condiciones de empleo y en particular el tamaño de las clases permitan que los docentes puedan enseñar en formas que permitan el desarrollo del conocimiento y las competencias necesarias en la era digital; y desarrollar una estrategia institucional práctica y coherente para dar soporte al tipo de enseñanza requerido en la era digital.

Hay una frase, muy significativa, con la que este autor, concluye su análisis sobre el «soporte de los profesores en la era digital», así señala que «dependerá de la imaginación de los docentes proponer nuevas formas de enseñanza para formar al tipo de profesional que necesitará el mundo en el futuro». Y razón no le falta, aunque, entendemos que, para el desarrollo de esa imaginación del docente en su enseñanza focalizado en la docencia virtual, es primordial que éste se encuentre dotado de las herramientas y conocimientos que le permitan llegar a dicho punto.

\section{Reflexiones.}

A modo de conclusiones, la formación en docencia virtual, en toda su extensión y en sus diferentes variables, del profesorado universitario se instituye en la actualidad en una acuciante necesidad.

La docencia virtual, ya presente en nuestras Universidades, se ha tornado en eje principal con la llegada de la Covid 19 para la impartición de las enseñanzas en todos los ámbitos de la misma y ello requiere la adquisición de los conocimientos, habilidades y competencias que posibiliten mantener la calidad docente, lo que, en ocasiones, supone la previa y adecuada formación en materia digital del profesorado, pasando éste, como se decía, de impartir enseñanza a recibirla para, valga la redundancia, poder continuar impartiendo la propia docencia adecuada al nuevo escenario. Un escenario que, sin embargo, tampoco puede decirse que fuese desconocido y en el que ya se venía trabajando pero que ese protagonismo que adquiere requiere un esfuerzo por parte de todos.

Y si la formación del docente es esencial, de igual forma lo es adquirir y saber desarrollar otras competencias que casi aparecen inherentes en un escenario de docencia total o mayoritariamente virtual. Aspectos como la prevención del abandono, la atención al estudiante o la motivación, se instituyen a su vez en factores claves a los que se ha de prestar particular atención. 
A día de hoy para poder ejercitar la función docente, en los términos previstos en la propia LOUA, es imprescindible intensificar esa formación docente a la que ya se hace referencia en el meritado cuerpo legal, lo que supone que, a las convencionales funciones docentes, investigadoras y de gestión del profesorado se sume una cuarta: la formación.

Es preciso una firme apuesta desde las instituciones (como ya se viene haciendo en la práctica totalidad de Universidades) por facilitar y proveer de los medios que posibiliten esa puesta al día del profesorado para hacer frente al escenario actual que se presenta ya como un escenario que en adelante se alzará en protagonista en las enseñanzas universitarias.

\section{Bibliografía.}

- AREA MOREIRA, M. (2018) "De la enseñanza presencial a la docencia digital. Autobiografía de una historia de vida docente". RED. Revista de Educación a Distancia, núm. 56, art.1, págs..1-21. Disponible: http://dx.doi.org/10.6018/red/56/1

- AREA MOREIRA, M., SAN NICOLÁS SANTOS, M.B. Y SANABRIA MESA, L. (2018) "Las aulas virtuales en la docencia de una universidad presencial: la visión del alumnado". Revista Iberoamericana de Educación a Distancia, 21(2), págs. 179198. Disponible: http://dx.doi.org/10.5944/ried.21.2.20666

- CALDERÓN PATIER, C. y GONZÁLEZ LORENTE, Á. (2009) “El papel del profesor universitario español en el EEES". Disponible: https://www.uv.es/motiva2/Ponencias\%20Motiva2009/docs/53.pdf

- DURÁN RODRÍGUEZ, R. y ESTAY-NICULCAR, C.A. (2016) "Las buenas prácticas docentes en la educación virtual universitaria". REDU, Revista de docencia universitaria, vol. 14 (2), julio-diciembre, págs. 159-186. Disponible: https://polipapers.upv.es/index.php/REDU/issue/view/638

- FROILÁN, J. Y GISBERT, M. (2012) "El cambio organizacional en la universidad a través del uso de los campus virtuales desde la perspectiva de los estudiantes". Pixel-Bit, Revista de Medios y Educación, núm. 40, págs. 75-88.

- GARCÍA ARETIO, L. (2013) "Fundamento y Componentes de la Educación a Distancia”. RIED. Revista Iberoamericana de educación a distancia, 2 (2).

- GARCÍA ARETIO, L. (2019) "El problema del abandono en estudios a distancias. Respuestas desde el Diálogo Didáctico Mediano". RIED, Revista Iberoamericana de Educación a Distancia, Asociación Iberoamericana de Educación Superior a Distancia, vol. 22, núm. 1. Disponible: https://doi.org/10.5944/ried.22.1.22433

- PIRIZ DURÁN, S. (2015) UNIVERSITIC 2015. “Análisis de las TIC en las Universidades Españolas". Madrid: Crue Universidades Españolas. Disponible: http://www.crue.org/Documentos\%20 
- URBINA, S. y SALINAS, J. (2014) "Campus virtuales: una perspectiva educativa y tendencias". RED. Revista de Educación a Distancia, núm. 42, septiembrediciembre, págs. 6-21. Disponible: https://www.redalyc.org/pdf/547/54731828002.pdf

- SÁNCHEZ SANTAMARÍA, J. Y MORALES CALVO, S. (2012) "Docencia universitaria con apoyo en entornos virtuales de aprendizaje (EVA)". Digital Education Review, núm. 21, págs. 33-46. Disponible: http://greav.ub.edu/der/

- TINTAYA, A.E. (2009) "Desafíos y fundamentos de educación virtual”. Buenos Aires, El Cid Editor.

- TONY BATES, A.W. (2017) "La enseñanza en la Era Digital. Una guía para la enseñanza y el aprendizaje". Obra publicada bajo licencia internacional de propiedad Creative Commnos no comercial.

- VARELA-OROL, C. (2011) “¿Hacia un nuevo paradigma bibliotecario? El nuevo orden digital". El profesional de la información, 20 (5), págs. 564-570. 